\title{
Private sector costing of voluntary medical male circumcision in South Africa
}

Michel Tchuenche

Steven Forsythe

Follow this and additional works at: https://knowledgecommons.popcouncil.org/departments_sbsr-hiv

Part of the Demography, Population, and Ecology Commons, Family, Life Course, and Society Commons, International Public Health Commons, and the Medicine and Health Commons How does access to this work benefit you? Let us know!

\section{Recommended Citation}

Tchuenche, Michel and Steven Forsythe. 2017. "Private sector costing of voluntary medical male circumcision in South Africa," Project SOAR Final Report. Washington, DC: Population Council. 


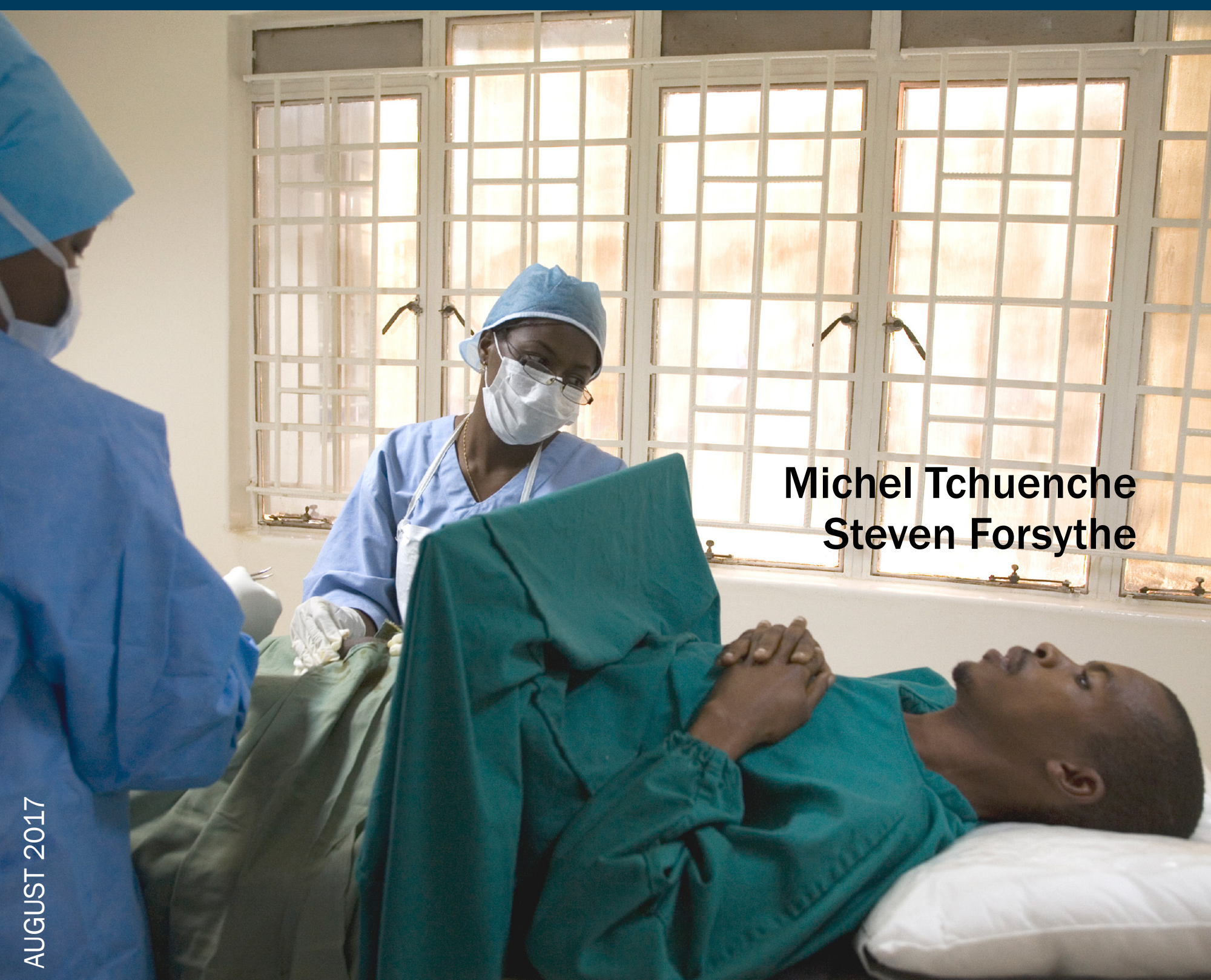





\section{Private Sector Costing of Voluntary Medical Male Circumcision in South Africa}

Avenir Health

Michel Tchuenche

Steven Forsythe 
Project SOAR

Population Council

4301 Connecticut Ave, NW, Suite 280

Washington, D.C. 20008 USA

Tel: +1202 2379400

Fax: +1 2022378410

projsoar.org

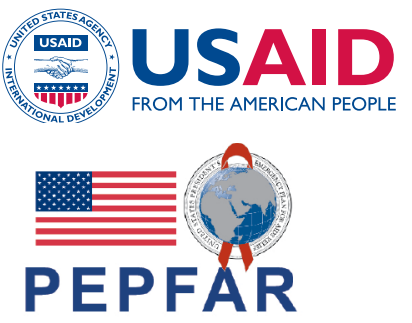

Project SOAR (Cooperative Agreement AID-OAA-A-14-00060) is made possible by the generous support of the American people through the President's Emergency Plan for AIDS Relief and the United States Agency for International Development (USAID). The contents of this guidance document are the sole responsibility of Project SOAR and the Population Council and do not necessarily reflect the views of USAID or the United States Government.

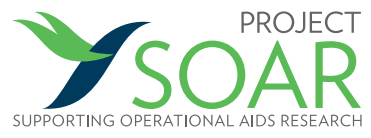

Through operations research, Project SOAR will determine how best to address challenges and gaps that remain in the delivery of HIV and AIDS care and support, treatment, and prevention services. Project SOAR will produce a large, multifaceted body of high-quality evidence to guide the planning and implementation of HIV and AIDS programs and policies. Led by the Population Council, Project SOAR is implemented in collaboration with Avenir Health, Elizabeth Glaser Pediatric AIDS Foundation, Johns Hopkins University, Palladium, and The University of North Carolina.

POPULATION COUNCIL

Ideas. Evidence. Impact.
The Population Council confronts critical health and development issues-from stopping the spread of HIV to improving reproductive health and ensuring that young people lead full and productive lives. Through biomedical, social science and public health research in about 50 countries, the Council works with our partners to deliver solutions that lead to more effective policies, programs, and technologies to improve lives worldwide. Established in 1952 and headquartered in New York, the Council is a nongovernmental, nonprofit organization with an international board of trustees.

Avenir Health was founded in 2006 as a global health organization that works to enhance social and economic development by providing tools and technical assistance in policy, planning, resource allocation and evaluation. Avenir Health focuses on developing and implementing demographic, epidemiological and costing models for long-range planning to assist with setting goals, strategies, and objectives. Avenir Health assists in both developing and implementing programs in HIV/AIDS, reproductive health, maternal health and other programming areas, working with government agencies, foundations, corporations, and nongovernmental organizations around the world.

Front cover photo credit: Richard Lord, courtesy of the Population Council.

Published in August 2017. @2017 The Population Council, Inc.

Suggested citation: Tchuenche, Michel and Steven Forsythe. 2017. "Private sector costing of voluntary medical male circumcision in South Africa," Project SOAR Final Report. Washington, D.C.: Population Council. 


\section{TABLE OF CONTENTS}

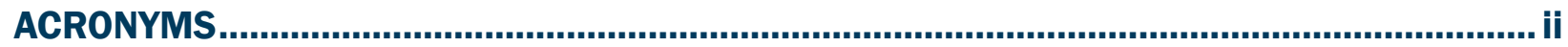

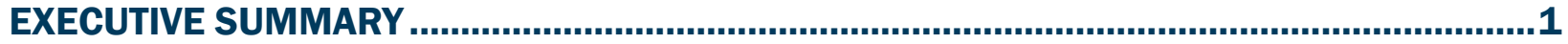

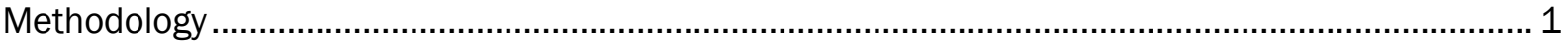

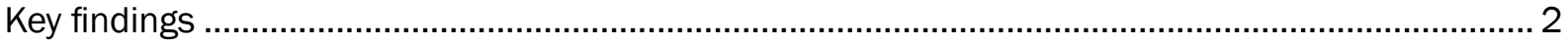

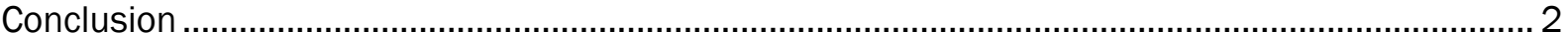

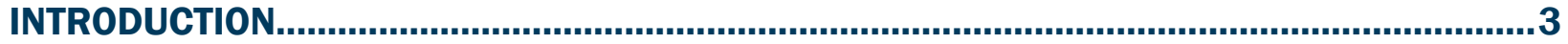

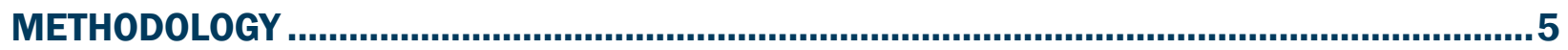

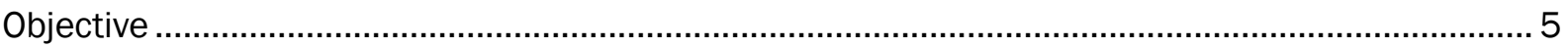

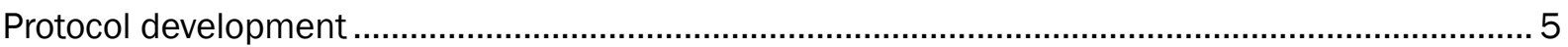

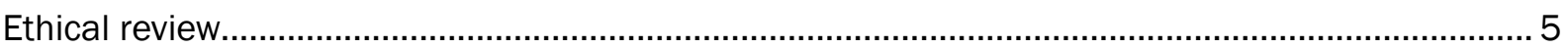

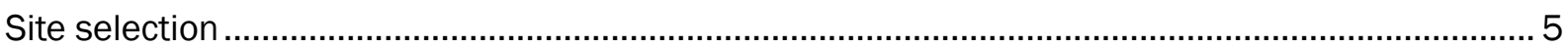

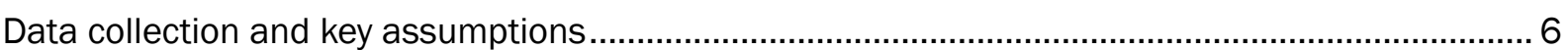

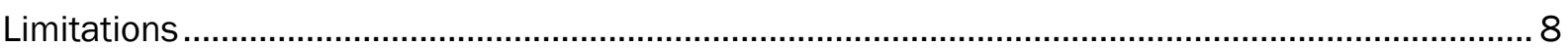

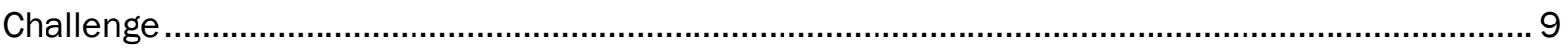

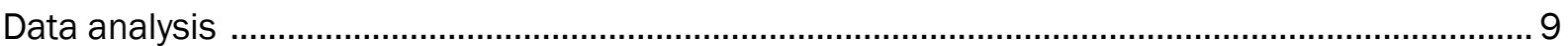

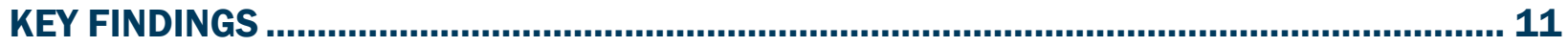

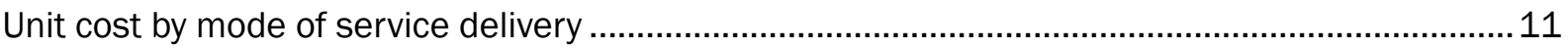

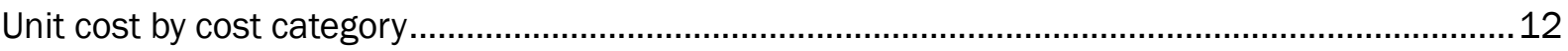

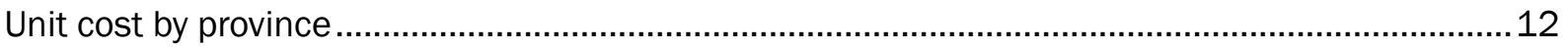

Unit cost in urban, peri-urban, and rural sites ....................................................................... 12

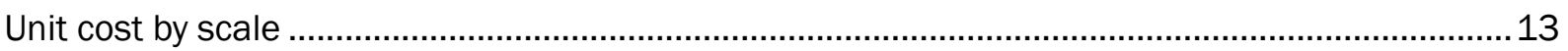

CONCLUSION AND RECOMMENDATIONS............................................................................... 15

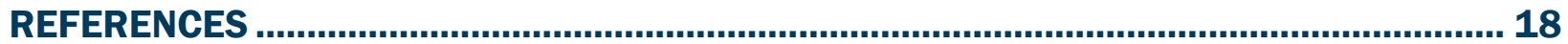




\section{ACRONYMS}

$\begin{array}{ll}\text { AIDS } & \text { Acquired immune deficiency syndrome } \\ \text { CQI } & \text { Continuous quality improvement } \\ \text { DMPPT } & \text { Decision Makers' Program Planning Toolkit } \\ \text { HIV } & \text { Human immunodeficiency virus } \\ \text { HPP } & \text { Health Policy Project } \\ \text { NDoH } & \text { National Department of Health (South Africa) } \\ \text { PEPFAR } & \text { U.S. President's Emergency Plan for AIDS Relief } \\ \text { SOAR } & \text { Supporting Operational AIDS Research } \\ \text { UNAIDS } & \text { Joint United Nations Programme on HIV/AIDS } \\ \text { URC } & \text { University Research Co., LLC } \\ \text { USAID } & \text { U.S. Agency for International Development } \\ \text { VMMC } & \text { Voluntary medical male circumcision } \\ \text { WHO } & \text { World Health Organization }\end{array}$




\section{EXECUTIVE SUMMARY}

South Africa has the largest population of people living with HIV in the world. In 2010, after voluntary medical male circumcision had been shown to be one of the most cost-effective strategies for preventing HIV infections, South Africa initiated a voluntary medical male circumcision (VMMC) program as a part of the country's HIV prevention strategy. In 2012 the National Department of Health $(\mathrm{NDoH})$ set an ambitious target of performing 4.3 million circumcisions by 2016 (NDoH 2012).

At the request of and in collaboration with the $\mathrm{NDoH}$, a detailed study of the costs of providing VMMC in South Africa at government and PEPFAR-supported (U.S. President's Emergency Plan for AIDS Relief) urban, rural, and peri-urban VMMC facilities was conducted in 2015. This study of 33 sites noted that the lack of data from the private sector represented a gap in knowledge concerning the overall cost of scaling up services in South Africa (Tchuenche et al. 2017). Because of the NDoH's interest in expanding coverage to reach its VMMC target, either within private or public sector facilities, Project SOAR-Supporting Operational AIDS Research-was requested to conduct a private-sector costing of providing VMMC services in South Africa to address this limitation. Therefore, the objective of this study was to derive the private provider unit cost of delivering medical male circumcision in South Africa.

The findings presented in this report provide a detailed investigation, through a comprehensive bottom-up approach, of the costs to private providers in offering VMMC to clients.

\section{METHODOLOGY}

Three provinces-Gauteng, KwaZulu-Natal, and Mpumalanga-were purposively selected out of the nine provinces of South Africa. Through an extensive stakeholder engagement process necessary to obtain both provincial and private sector buy-in and support for the study, 13 facilities were eventually selected, mainly based on their willingness to participate in the study. Financial and human resource data required for estimating the unit cost per VMMC beneficiary in the private sector were collected retrospectively from July to November 2016, covering the most recent 12-month period of 2015/2016 (for most facilities, this was June 2015 to May 2016). Key VMMC personnel interviews were semi-structured and directed at facility VMMC program managers, finance officers, or the general practitioner/medical officers conducting the surgical procedure. Unit costs were calculated using a bottom-up approach by cost drivers (direct labor, consumables, indirect labor, overhead, training, equipment, and vehicles), geographic location (province) and level of urbanization (urban, peri-urban, or rural). Since the private facilities do not themselves conduct training and continuous quality improvement (CQI), these cost elements were collected from government and PEPFAR-supported programs. 


\section{KEY FINDINGS}

This study determined that the unit cost (including the cost of training and CQI) to provide VMMC at private facilities was R1,487. Cost drivers were largely consumables (41 percent) and direct labor (34 percent). Other components of unit costs were CQI (13 percent), overhead and support staff (4 percent each), training ( 2 percent), and equipment and vehicle/transport (1 percent each).

Mpumalanga had a total of three sites in the study sample: two rural and one urban facilities and was the province with the highest unit cost $(R 1,588)$, followed by Gauteng with seven sites $(R 1,495)$ and Kwazulu-Natal with four sites (R1,226). Eleven out of the 13 surveyed facilities were fixed sites $(R 1,541)$, while the remaining two were outreach $(R 1,685)$ and a fixed site with outreach and mobile services (R1,327). Surveyed facilities were classified depending on their level of urbanization: urban, peri-urban, or rural. The unit cost was R1,531 at the seven surveyed urban sites, R1,399 at the four peri-urban sites, and R1,552 at the two rural sites.

\section{CONCLUSION}

Contrary to popular belief that private sector operational costs differ significantly from those incurred in the public sector, this study found that the average unit cost of providing VMMC in private facilities $(R 1,487)$ is not fundamentally different from the unit cost of providing the same service in government and PEPFAR-supported facilities (R1,431) (Tchuenche et al. 2017). The largest components of this unit cost (across all facilities sampled) are consumables and direct labor, which is in agreement with other costing studies of VMMC in sub-Saharan Africa (Bollinger et al. 2014). It is expected that results from this study will provide health policy and decision makers as well as VMMC programme managers at the national, provincial, and district levels with valuable costing data necessary to make informed decisions regarding the funding and scale-up of VMMC in South Africa in partnership with the private sector.

Results from this study could inform discussion with private insurance providers in South Africa about standardization VMMC tariffs. It also provides a strong rationale for reimbursing private sector providers for circumcisions of uninsured clients. 


\section{INTRODUCTION}

Since the mid-2000s, voluntary medical male circumcision (VMMC) has been shown to be a promising HIV prevention strategy and one of the most cost-effective of those available for preventing new HIV infections (Njeuhmeli et al. 2011, Siegfried et al. 2009). This led the Joint United Nations Programme on HIV/AIDS (UNAIDS) and the World Health Organization (WHO) to identify 14 priority countries in eastern and southern Africa (including South Africa) for VMMC scale-up (UNAIDS 2011, WHO 2015). On the basis of the overwhelming evidence and the fact that the VMMC program in South Africa-the country with the largest HIV epidemic of any country in the world (UNICEF Undated)-has tremendous potential to make a significant public health impact by preventing large numbers of new HIV infections and saving countless lives (National Department of Health [NDoH] 2012), in 2010 the South African Government introduced the procedure as an HIV prevention intervention (South African National AIDS Committee 2011).

About 46 percent of males over age 15 in South Africa have been circumcised, through either a traditional or a medical procedure. However, only 19 percent have been circumcised medically (Shisana et al. 2014, Govender et al. 2014). South Africa has set a target of circumcising 80 percent of HIV-negative males between the ages of 15 and 49 (NDoH 2012). To achieve this ambitious target would have required circumcising 4.3 million men and boys between 2010 and 2016 (NDoH 2012); this represents the largest number of men to be circumcised in a single country in the world (UNICEF Undated).

Larson et al. (2015) compared the costs of facility-based and mobile VMMC service delivery in Uganda. The major limitation of this study is its narrow focus on data only from the Makerere University Walter Reed VMMC program and the lack of comparable data from the private sector. Kripke et al. (2014) explored the impact of scaling up VMMC in the context of the 2020 Joint United Nations Programme on HIV/AIDS (UNAIDS) 90-90-90 targets for treatment (90 percent of all those living with HIV are diagnosed, 90 percent of those diagnosed are on antiretroviral treatment (ART), and 90 percent of those on ART are virally suppressed by 2020). Using a mathematical model-the Decision-Makers' Program Planning Toolkit (DMPPT) 2 (Kripke et al. 2016)-the authors showed that through reaching 80 percent VMMC coverage by 2015 and sustaining this through 2025, South Africa could avert 94,000 new HIV infections. However, by early 2015, the South African VMMC program had performed less than 50 percent of its target of 4.3 million male circumcisions by 2016 (only about 1.8 million VMMCs, or 43 percent of its target, has been achieved) (Larson et al. 2015). Consequently, as the country is lagging behind on achieving its VMMC target, the government seeks to explore other service provision modalities, such as partnering with the private sector. Another rationale for engaging with the private sector is the shortage of medical doctors in the public sector. Indeed, Bertrand et al. (2013) noted that medical doctors in public facilities are much more likely than their counterparts in other services to burn out and leave the VMMC program. Therefore harnessing the human resources in the private sector would contribute to mitigating the impact of VMMC staff turnover in the government-supported facilities. Thus, private sector providers represent a strategic potential 
to increase access to VMMC services, but may also draw clients who may not necessarily go to public health facilities for services. Given that the NDoH seeks to involve the private sector in reaching its VMMC targets, estimating the private sector unit cost of providing VMMC services will complement the first phase of this study that estimated the cost of providing VMMC at government and PEPFAR-supported (U.S. President's Emergency Plan for AIDS Relief) urban, rural, and peri-urban facilities (Tchuenche et al. 2017). 


\section{METHODOLOGY}

\section{OBJECTIVE}

The objective of this study was to derive the unit cost of delivering VMMC at private sector facilities in South Africa. This objective was established by the NDoH and by PEPFAR due to the perceived need to better understand the resources that would be required to further scale up the provision of VMMC services in South Africa's private sector.

\section{PROTOCOL DEVELOPMENT}

The study protocol and facility data collection instruments were developed in 2014 by the Health Policy Project (HPP) research team, with support from the NDoH and key stakeholders. This original protocol was developed with the intention of collecting cost data from public and private facilities. At the time, the project was not successful at collecting cost data from private sector facilities. The original protocol was amended by Project SOAR (Supporting Operational AIDS Research) in consultation with the NDoH and PEPFAR and stipulated that Project SOAR would collect necessary data at private facilities in three selected provinces-Gauteng, KwaZulu-Natal, and Mpumalanga. Project SOAR did not modify the original facility data collection instrument and costing tool developed by HPP for the government and PEPFAR-supported facilities costing (Tchuenche et al. 2015).

\section{ETHICAL REVIEW}

A final five-year ethics clearance certificate was received on 25 February 2015 from the South African Human Research Ethics Committee (Medical), University of Witwatersrand, South Africa. This ethical approval covered both the public sector costing (the previous study), and the private sector costing (the current study).

\section{SITE SELECTION}

Three provinces-Gauteng, KwaZulu-Natal, and Mpumalanga-were purposively selected in consultation with the NDoH and PEPFAR, with the aim of conducting data collection in at least three facilities in each of the three selected provinces. Primary selection factors were study resource limitations and stronger stakeholder engagement in these three study provinces. Thus, through an extensive stakeholder engagement process, necessary to obtain private sector buyin and support for the study, a list of VMMC private service providers who had routinely provided VMMC services for at least six months was compiled in each of the three provinces. Most facilities approached-20 in total-were willing to participate in this study. Eventually, 13 facilities agreed 
to provide data. These facilities were classified based on their geographic location: urban, peri-urban, or rural setting, as well as mode of service delivery: fixed, outreach, and fixed with outreach and mobile services. Because of confidentiality, the names of the facilities will not be included in this report. Table 1 provides the service delivery mode, location, and total number of VMMC clients at each of the surveyed facilities.

\begin{tabular}{|c|c|c|c|c|}
\hline Province & Facility code & Service delivery model & Location & $\begin{array}{l}\text { Total \# of VMMC } \\
\text { clients (annual) }\end{array}$ \\
\hline \multirow[t]{6}{*}{ Gauteng } & G1 & Fixed & Urban & 4,949 \\
\hline & G2 & Fixed & Urban & 2,678 \\
\hline & G3 & Fixed & Peri-urban & 1,758 \\
\hline & G4 & Fixed & Peri-urban & 1,235 \\
\hline & G5 & Fixed & Peri-urban & 8,378 \\
\hline & G6 & Fixed, outreach \& mobile & Urban & 1,080 \\
\hline \multirow[t]{4}{*}{ KwaZulu-Natal } & K1 & Fixed & Peri-urban & 216 \\
\hline & $\mathrm{K} 2$ & Fixed & Urban & 1,570 \\
\hline & K3 & Outreach & Urban & 575 \\
\hline & K4 & Fixed & Urban & 1,300 \\
\hline \multirow[t]{3}{*}{ Mpumalanga } & M1 & Fixed & Rural & 3,765 \\
\hline & M2 & Fixed & Rural & 2,770 \\
\hline & M3 & Fixed & Rural & 1,217 \\
\hline
\end{tabular}

\section{DATA COLLECTION AND KEY ASSUMPTIONS}

Financial expenditure and human resource data required for estimating unit cost per VMMC beneficiary in the private sector were collected retrospectively between June and November 2016 from 13 selected facilities across three of the nine provinces of South Africa, covering the most recent 12-month period in 2015/16 for which program data were available (for most facilities, this was June 2015 to May 2016). Key VMMC personnel interviews were semi-structured and directed at facility VMMC program managers, finance officers, or the general practitioner/medical officers conducting the surgical procedure.

\section{Human resources}

At each facility, information was collected about direct and indirect staff; direct staff members were largely clinical staff, such as general practitioners, nurses, and counselors. Shared staff provide direct services, while indirect staff members provide support services only and do not provide any direct VMMC services to clients.

Information on the employment status (permanent versus contracted staff) was collected for both direct and indirect staff members. Additional information collected included the number of personnel, their salaries, and the percentage of time allocated to VMMC. For VMMC-only sites, the direct and support staff level of effort to provide VMMC services was 100 percent. 


\section{Medications and other consumables}

Information was collected on the percentage of VMMC clients that received specific types of medicines/consumables, the quantity distributed to each VMMC client, and the cost of each item. Priority was given to sites that provided their own estimates of input costs. However, in cases where costs of medications and/or consumables were not available, the study team used the information collected from a variety of sources in 2015, including the Supply Chain Management System, Clinton Health Access Initiative, and PEPFAR as well as from Northdale Hospital and the voluntary medical male circumcision Centre of Excellence in KwaZulu-Natal (Tchuenche et al. 2017). Although some facilities indicated they do not purchase the circumcision kits from PEPFAR, the PEPFAR-provided cost of R193 per kit was attributed.

\section{Equipment and furniture}

Information about equipment and furniture was obtained from each site. Facilities were asked to provide a list of all equipment and furniture utilized in the screening/review room, the counseling area, the operating theatre, and/or any relevant sterilization areas. In addition, facilities were asked to identify general equipment that was used as part of the VMMC program. Each facility then provided information on the number of items utilized and the estimated percentage of time equipment/furniture was utilized for VMMC. The useful life of equipment and furniture was obtained from WHO-CHOICE (Choosing Interventions that are Cost-Effective) (2014). When useful life estimates were not available from WHO-CHOICE, data were collected from a recent circumcision costing study at government and PEPFAR-supported facilities in South Africa (Tchuenche et al. 2017); when the latter was not available from WHO-CHOICE, capital costs were allocated over the useful life of equipment or furniture without discounting.

\section{Vehicles}

Vehicles were used for transportation of supplies and staff to VMMC outreach sites and very rarely for the transportation of clients. For facilities offering other services besides male circumcision, only vehicles used by the facility as part of the VMMC program were recorded as well as the proportion of time the vehicles were used by the VMMC program. Vehicle costs were amortized and the proportion allocated to the VMMC program was determined based on the number of VMMC clients relative to the total number of clients at the facility.

\section{Overhead}

Annual overhead costs included a range of items-costs associated with utilities (water, electricity, internet, telephone, waste management, cleaning services, etc.) and the rental cost of the facility. Overhead costs were apportioned to the VMMC program based on the number of VMMC clients relative to the total number of clients seen at the facility.

\section{Continuous quality improvement (CQI)}

Given that the $\mathrm{NDoH}$ seeks to involve the private sector in reaching its ambitious VMMC targets, it is anticipated that CQI will be extended to VMMC private providers. For this reason, average cost of R191 (Tchuenche et al. 2017) to provide CQI for PEPFAR partners in South Africa was added to the calculated VMMC unit cost. 


\section{Training}

Training costs were provided in KwaZulu-Natal only, but this information was insufficient to extrapolate to the other two provinces. The study team instead used the average national-level data on the cost of training per circumcision of R25 that was obtained from implementing partners that support the training of service providers at the national and district levels (Tchuenche et al. 2017).

\section{Other key assumptions}

This study is a detailed economic cost analysis that considered all costs, except demand creation and societal costs. In order to estimate the private sector unit cost of providing VMMC services, key assumptions were made:

- To compare the private and public (government and PEPFAR-supported sites) sectors' unit costs of providing VMMC services, CQI and training costs obtained from the costing of VMMC at government and PEPFAR-supported facilities in South Africa (Tchuenche et al. 2017) were included.

- The proportion of VMMC clients relative to the total client volume at the facility was used to apportion indirect staff time and other overhead to MMC costs. Both facility and contextual data were collected to facilitate analysis and cost allocation.

\section{LIMITATIONS}

This costing study is not exhaustive and some limitations are noted below.

\section{Limited sample}

Data were only collected at 13 facilities from three of South Africa's nine provinces. A larger sample size-which might have been difficult to secure due to the challenges noted below-could have provided better opportunities to compare unit costs across all nine provinces.

\section{Changing costs and cost limitations}

This study did not identify how unit costs might change in the future as South Africa further scales up its program. On the one hand, unit costs might decline as facilities scale up and are able to efficiently utilize resources. Conversely, unit costs might increase as it becomes increasingly difficult to identify the diminishing number of clients who are ready and willing to adopt male circumcision.

\section{Effects of recall bias}

Study researchers did not do a time-motion analysis of how staff spent their time on the VMMC program. Instead, respondents were asked to provide a general allocation of time during each day. Since the allocation of time was based on recall and not on actual observations, it is possible that there may have been an overestimation or underestimation of time spent on male circumcision. 
In most facilities client volumes vary significantly from month to month and estimates of level of effort by service providers may be biased by recent experiences. Future studies would benefit from a minimum number of observations at each site to obtain more accurate estimates of time allocated to VMMC. Likewise, while every effort was made to obtain all information about resources used at facilities, the interview process required respondents to accurately recall and identify direct and indirect resources.

\section{Demand creation}

Demand creation is the process of mobilizing and motivating men to access VMMC services (Tchuenche et al. 2017). This is crucial for the successful implementation of a VMMC program. In some cases, private sector providers readily engaged in demand creation through newspaper adverts and other demand creation approaches. However, having private sector providers invest in demand creation is only likely to occur if they observe that the demand creation efforts are successful (increasing client flow) and if they have a financial incentive to expand their clientele. It was noted, for example, that some private providers engage in VMMC so that they can also offer clients other services and ideally assure that the client makes return visits for other health issues. Because this study did not link spending on demand creation to the increased number of clients, demand creation was excluded from the unit cost calculation.

\section{CHALLENGE}

The main challenge of this study was securing participation from the service providers. Most service providers were willing to share information initially, but some ultimately did not commit to interviews due to time constraints. Service providers recognized that they will lose income due to the nature of their business and the substantial time required for the interview process.

Despite this challenge, we were able to collect data from our target of 3 to 5 facilities in all three provinces. We believe that the costing data presented in this report will inform VMMC strategic planning in South Africa, as it provides VMMC policymakers with valuable information required to partner with the private sector in order to meet the ambitious target set by the government.

\section{DATA ANALYSIS}

Prior to analyzing the data, the study team conducted extensive site visits to interview facility staff members involved in the provision of VMMC services. Where records were incomplete, the research team requested clarifications. Following the completion of the data cleaning process, the Project SOAR research team entered data from the 13 facility surveys into the developed costing model: an Excel spreadsheet customized to calculate as a key output the unit cost of VMMC in South Africa (Health Policy Initiative 2010).

Unit costs were derived using an ingredients-based approach where all inputs are listed, their costs are collected, and the contribution of these costs to the overall cost is quantified (Health Policy Initiative 2010, Menon et al. 2014). Major cost categories in the unit cost calculation were: 
- Direct costs (drugs and supplies, other consumables, equipment, non-consumable supplies, and direct and indirect personnel)

- Indirect costs (capital, maintenance, electricity and fuel, other utilities, and support personnel).

Overhead costs (such as water, electricity, internet, telephone, waste management, cleaning services, etc.) and the rental value of the facility were assigned to the VMMC program based on the number of VMMC clients relative to the total number of clients seen at the facility.

Facilities unit costs were obtained by dividing the total cost of providing VMMC during the study period by the number of beneficiaries. Average unit costs were then calculated as the weighted average of the 13 facilities' unit cost. 


\section{KEY FINDINGS}

From the data collected, the cost per circumcision performed at the 13 private facilities was determined to be R1,487. This cost includes the average training cost (R25) as well as CQI cost per circumcision performed (R191) in government and PEPFAR-supported sites (Tchuenche et al. 2017). While the unit cost of providing the same service in government and PEPFAR-supported facilities was R1,431 (Tchuenche et al. 2017) , the unit cost difference at private and public facilities was not statistically significant $(p=0.177)$.

\section{UNIT COST BY MODE OF SERVICE DELIVERY}

Figure 1 shows the unit cost difference between the types of facility: 11 fixed sites (no outreach services), one outreach site, and one fixed site with both mobile and outreach components. However, with only one outreach site and one fixed site with both mobile and outreach services, it is not possible to draw any meaningful conclusion on the difference of unit cost by mode of service delivery in the private sector.

\section{Figure 1 Unit costs by service delivery mode}

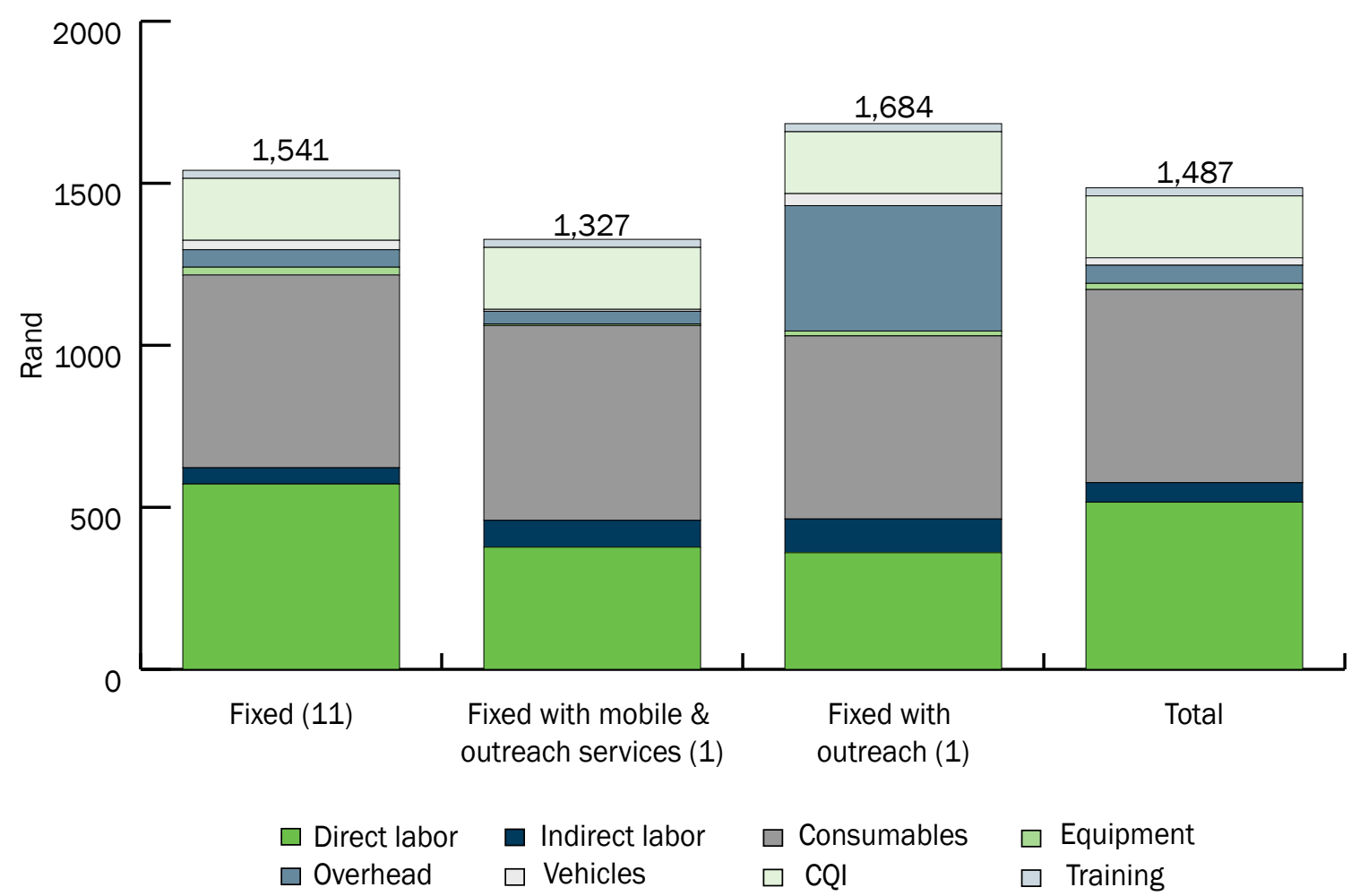




\section{UNIT COST BY COST CATEGORY}

Figure 2 indicates the major cost drivers for the average sample unit cost. The two largest components of total unit costs was consumables (40 percent), followed by direct labor (35 percent) which was comparable across both studies (accounting respectively for 75 percent of all costs at for-profit facilities, and 67 percent at public and nonprofit facilities) (Tchuenche et al. 2017). This result is in agreement with earlier studies, which found that the two main cost drivers associated with providing VMMC in sub-Saharan Africa are personnel and consumables (Bollinger et al. 2014). It is important to note that consumables and personnel cost were also the main cost drivers across all the facilities.

\section{Figure 2 Unit cost by major cost category}

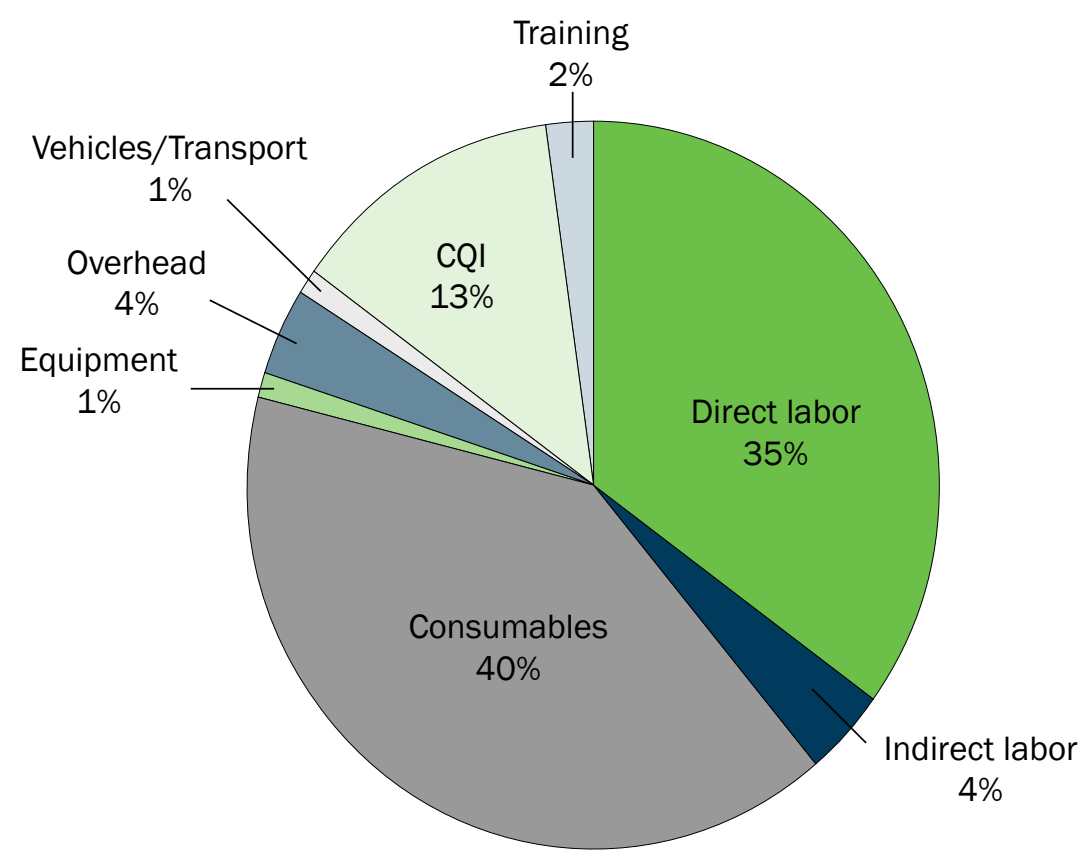

\section{UNIT COST BY PROVINCE}

Figure 3 provides a breakdown of unit costs by province. The number of MMC sites within a given province is indicated in brackets next to the province's name: Gauteng has six sites, followed by KwaZulu-Natal with four sites, and Mpumalanga with three sites. Mpumalanga, with two rural sites and one urban site in the sample, had the highest unit cost, followed by Gauteng and Kwazulu-Natal. The unit cost in Mpumalanga was driven by higher unit costs for labor (R747 vs R448 in KwaZulu-Natal and R401 in Gauteng). In fact, these three sites had more contracted staff than other surveyed private facilities in Gauteng and KwaZulu-Natal. Similar trends were observed at government and PEPFAR-supported facilities (Tchuenche et al. 2017). Gauteng had the largest numbers of sites in the sample (six in total), and the unit cost in this province did not differ significantly from the overall unit cost. 
Figure 3 Unit cost by province

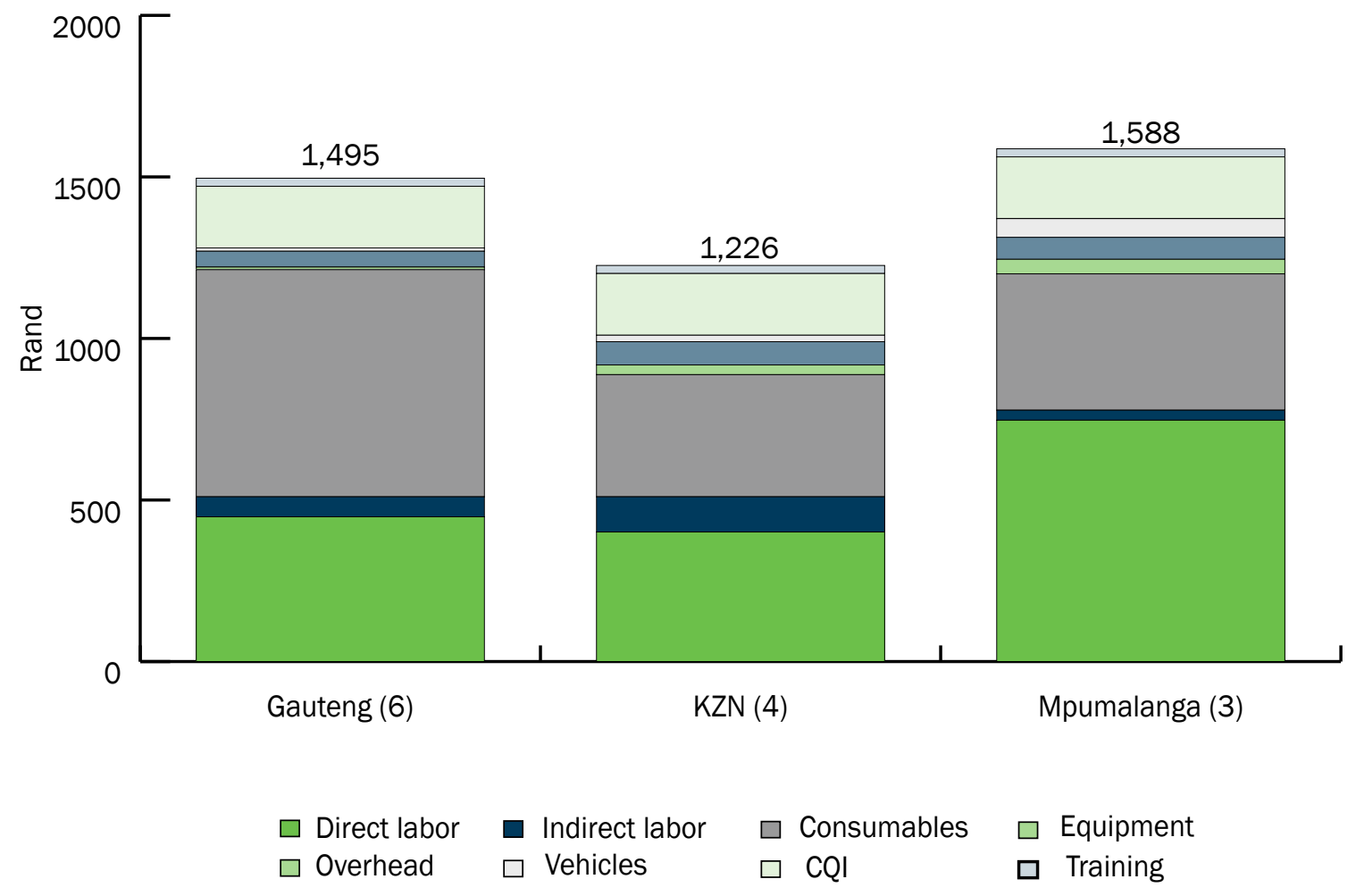

Figure 4 compares the components of the unit cost between the private and the public sectors. Direct labor represented the largest unit cost component in public facilities (43 percent), followed by consumables (24 percent), CQI (13 percent), indirect labor (11 percent), overhead (5 percent), training and equipment ( 2 percent), and vehicles (1 percent). One noticeable difference was the large amount of consumables used in private facilities (41 percent) versus in public facilities (24 percent).

This study found that the labor cost per circumcision performed at private facilities is R516, compared to R613 at public facilities in 2014 (Tchuenche et al. 2017). In other words, labor costs per circumcision at private facilities were not higher than they are in the public sector.

\section{UNIT COST IN URBAN, PERI-URBAN, AND RURAL SITES}

Each site was classified depending on the level of urbanization in the communities where the facility operates: urban, peri-urban, or rural areas in the province. There were a total of seven urban sites, four peri-urban sites, and two rural sites. Mpumalanga, the province with the highest unit cost, had two rural sites and one urban site. Figure 5 indicates that the unit costs of performing medical male circumcision were respectively R1,531, R1,399, and R1,552 at urban, peri-urban, and rural facilities. 
Figure 4 Comparison of unit cost components: public vs private sectors

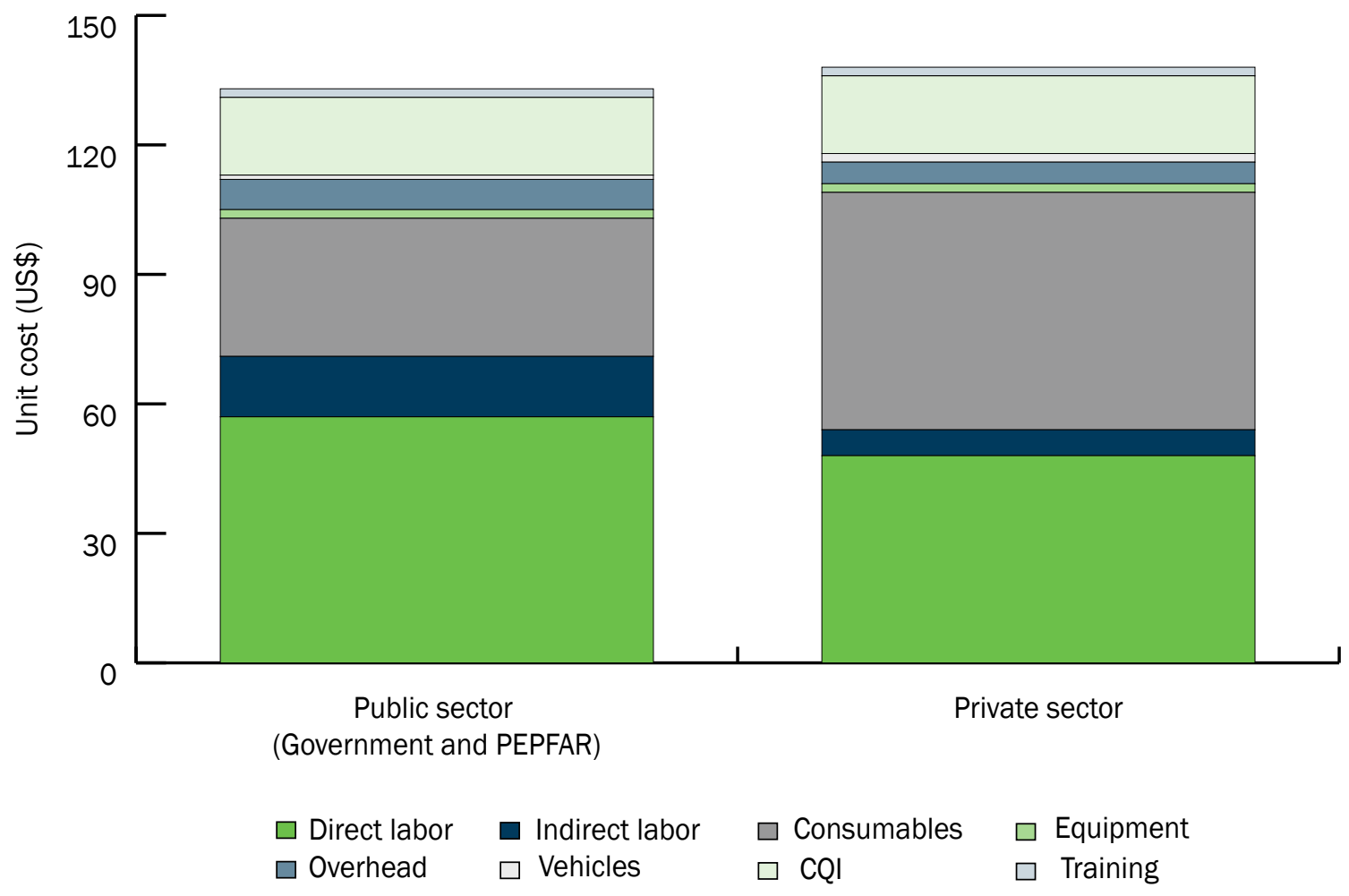

Figure 5 Unit cost by level of urbanization

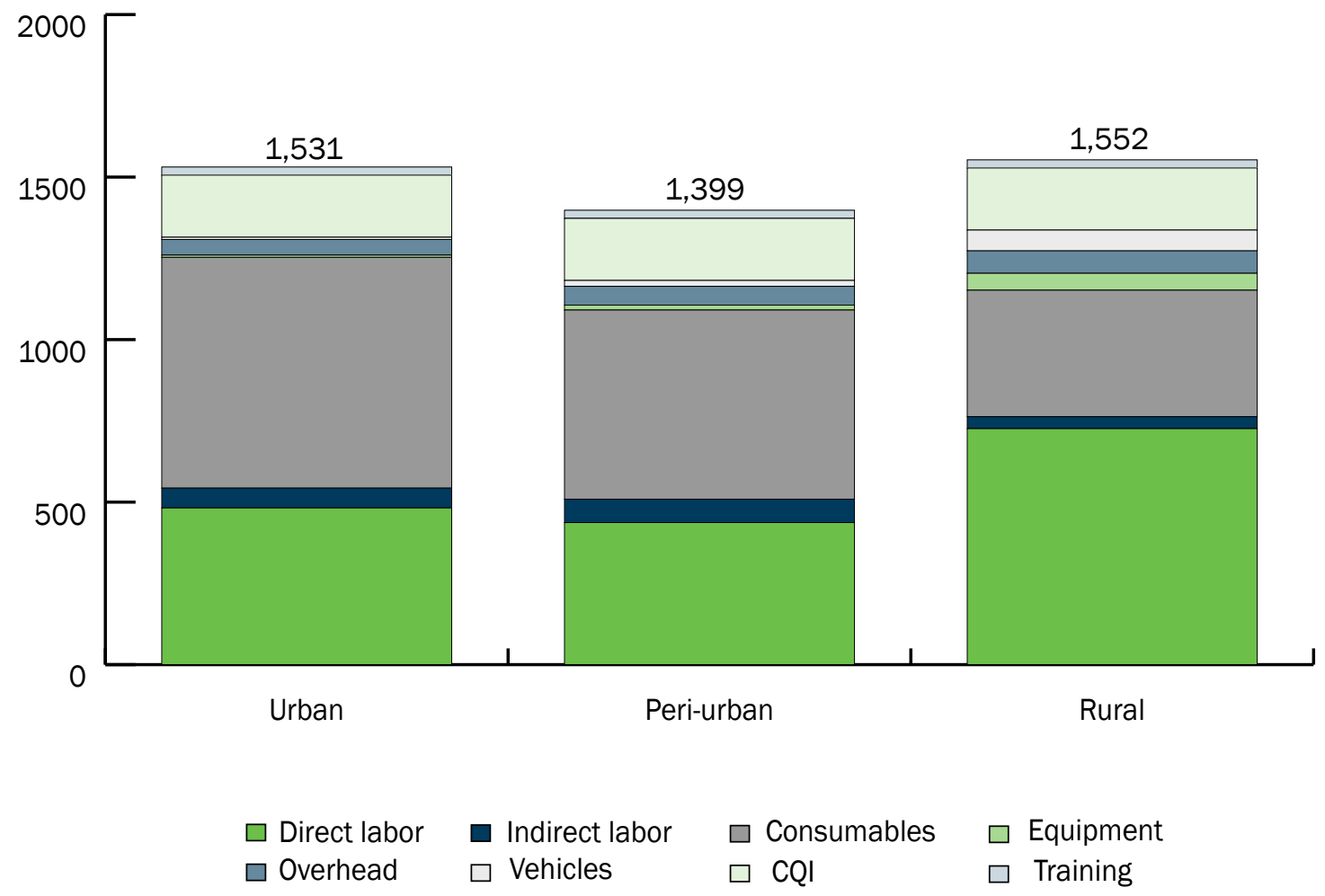




\section{UNIT COST BY SCALE}

Although scale may not fully explain variations in unit costs, the relationship between the number of circumcisions performed at private facilities and the average unit cost of each circumcision is explored in Figure 6. The blue line in Figure 6 shows that there is, as expected, an inverse relationship between volume and unit cost; sites which have higher numbers of MMC clients generally having a lower unit cost.

Over three-quarters (85 percent; 11/13) of sites performed more than 1,000 circumcisions annually. This is in contrast with the public sector where it was found that about 52 percent $(17 / 33)$ of all sites had more than 1,000 circumcisions per year (Tchuenche et al. 2017). The average number of circumcisions performed at government and PEPFAR-support facilities was 1,840 , while this study found that the average number of circumcisions performed annually at private facilities was 2,422 . The difference was not statistically significant $(p=0.204)$. The median number of clients was 22 percent higher in private facilities $(1,570)$ as compared to public facilities $(1,229)$. The higher volume in private facilities may be a factor in reducing the overall unit cost at private facilities.

Figure 6 Unit cost by scale

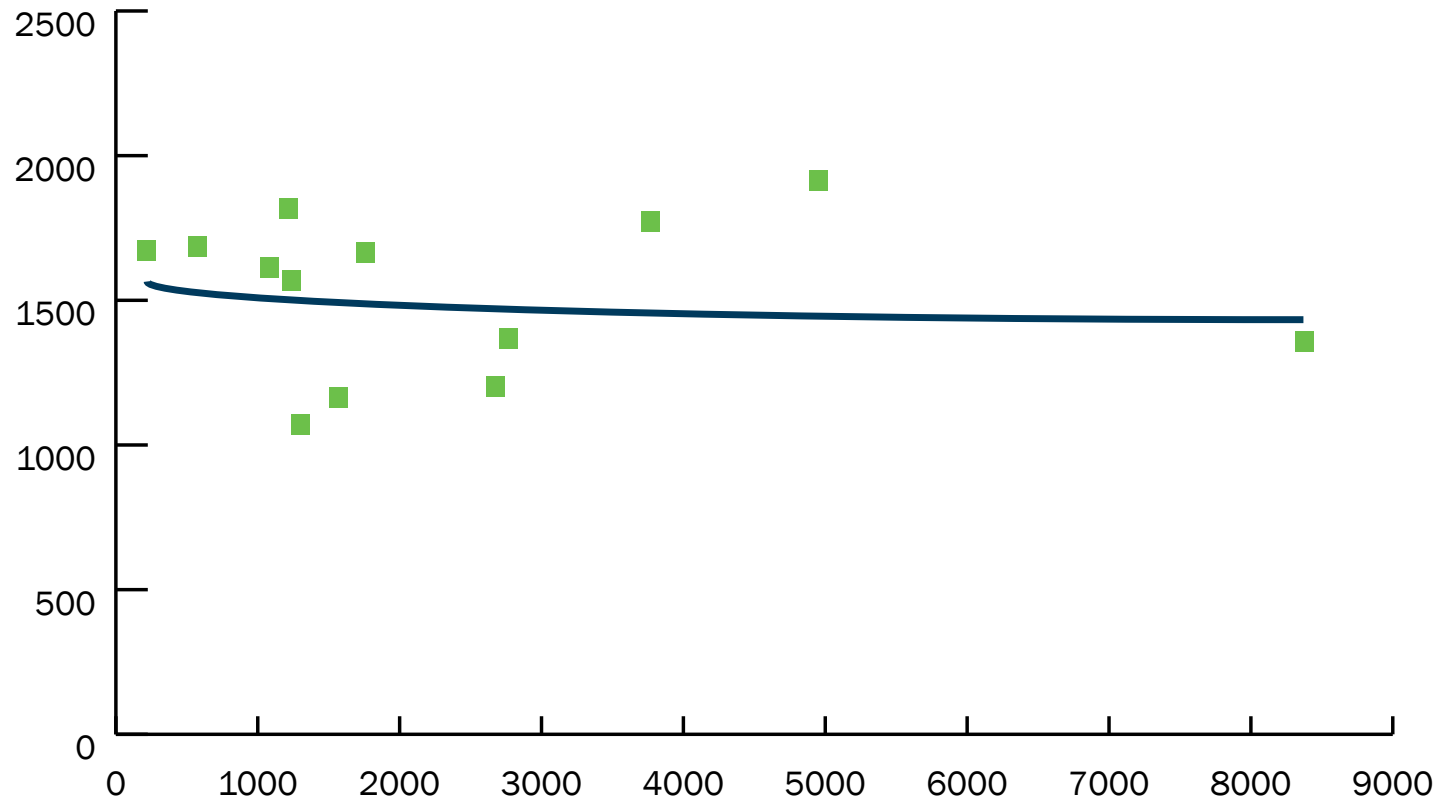




\section{CONCLUSION AND RECOMMENDATIONS}

Contrary to popular belief that private sector operational costs differ significantly from those incurred in the public sector, this study found that the unit cost of providing VMMC in private facilities $(R 1,487)$ was not fundamentally different from the unit cost of providing the same service in government and PEPFAR-supported facilities (R1,431) (Tchuenche et al. 2017). The unit cost difference was not statistically significant. The largest components of this unit cost were consumables and direct labor and were comparable across both studies (accounting respectively for 75 percent of all costs at for-profit facilities and 67 percent at public and nonprofit facilities (Tchuenche et al. 2017). This result is consistent with other costing studies that showed personnel and consumables are the two main cost drivers of providing VMMC in sub-Saharan Africa (Bollinger et al. 2014).

Private facilities noted that demand creation is critical to bringing more clients into their practice. However, the challenge they face is financing this demand creation and ensuring that patients get safe transport from home to clinic and back. About one-third of the facilities surveyed indicated that they spent some funds on demand creation, but they recruit community mobilizers only on a contract basis as the need arises. If the public sector can work synergistically with the private sector to better assess their demand creation needs, it should be feasible to further increase demand for private sector VMMC services without significantly increasing the cost of service delivery as well as the overall cost of VMMC demand creation. However, for the reasons indicated above (service provision not adequately linked to demand generation activities), demand creation cost was not included in the unit cost calculation.

Results from this study provide guidance to health policymakers and VMMC program managers at the national, provincial, and district level to make informed decisions regarding the funding and scale-up of VMMC in South Africa in partnership with the private sector. This study could inform discussion with private insurance providers in South Africa to standardize VMMC tariffs and allow insured clients to be provided services, but have them paid for by their insurance companies. It also provides a strong rationale for reimbursing private sector providers for circumcisions of uninsured clients. Thus, the government of South Africa should consider utilizing the services of private VMMC providers in order to meet its ambitious target and to sustain the VMMC program in the long run. 


\section{REFERENCES}

Bertrand, J. T. et al. 2013. "Systematic monitoring of the voluntary medical male circumcision scale-up in Eastern and Southern Africa (SYMMACS): Final report of results from Kenya, South Africa, Tanzania and Zimbabwe." Baltimore, MD: USAID Project Search: Research to Prevention; 2013.

Bollinger L. et al. 2014. "Cost drivers for voluntary medical male circumcision using primary source data from Sub-Saharan Africa," PLoS ONE 9(5): e84701. doi: 10.1371/journal. pone.0084701.

Govender, K. et al. 2014. "Voluntary medical male circumcision in South Africa: challenges and opportunities," S Afr Health Rev (SAHR). 2013/14. Available: http://www.hst.org.za/sites/ default/files/Voluntary_MMC.pdf.

Health Policy Initiative. 2010. Costing workbook: NGO Health Center. Available: https://www. malecircumcision.org/resource/costing-workbook-ngo-health-center.

Kripke, K. et al. 2014. "Assessing progress, impact, and next steps in rolling out voluntary medical male circumcision for HIV prevention in 14 priority countries in Eastern and Southern Africa through 2014," PLoS ONE 11(7): e0158767. doi: 10.1371/journal.pone.0158767.

Kripke, K. et al. 2016. "Age targeting of voluntary medical male circumcision programs using the Decision Makers' Program Planning Toolkit (DMPPT) 2.0," PLoS ONE 11(7): e0156909. doi:10.1371/journal.pone.0156909.

Larson, B. et al. 2015. "How much does it cost to improve access to voluntary medical male circumcision among high-risk, low-income communities in Uganda?," PLoS ONE 10(3):e0119484.

Menon, V. et al. 2014. "Costs and impacts of scaling up voluntary medical male circumcision in Tanzania," PLoS ONE 9(5): e83925. doi: 10.1371/journal.pone.0083925.

National Department of Health (NDoH). 2012. Strategic plan for the scale up of medical male circumcision (MMC) in South Africa, 2012-2016. Pretoria, South Africa: NDoH.

Njeuhmeli, E. et al. 2011. "Voluntary medical male circumcision: modeling the impact and cost of expanding male circumcision for HIV prevention in eastern and southern Africa," PloS Medicine 8(11): e1001132. doi: 10.1371/journal.pmed.1001132.

Shisana, O. et al. 2014. "South African national HIV prevalence, incidence and behaviour survey, 2012." Cape Town, SA: HSRC Press. 
Siegfried, N. et al. 2009. "Male circumcision for prevention of heterosexual acquisition of HIV in men," The Cochrane Database of Systematic Reviews (2). doi: 10.1002/14651858.CD003362. pub2.

South African National AIDS Committee (SANANC). 2011. National Strategic Plan on HIV, STIs and TB 2012-2016. Pretoria, South Africa: SANAC.

Tchuenche, M. et al. 2015. "Estimating the provider and client costs of medical male circumcision in South Africa." Washington, DC: Futures Group, Health Policy Project. http://www. healthpolicyproject.com/index.cfm?ID=publications\&get=pubID\&pubID=557

Tchuenche, M. et al. 2017. "Correction: the cost of voluntary medical male circumcision in South Africa,” PLoS ONE 12(1): e0169710. doi:10.1371/journal.pone.0169710.

UNAIDS. 2015. "HIV and AIDS estimates." Available: http://www.unaids.org/en/regionscountries/ countries/southafrica/.

UNICEF. Undated. "Eastern and Southern Africa," accessed 12 July 2017. Available: https://www. unicef.org/esaro/5482_HIV_AIDS.html.

Uthman, OA et al. 2010. "Economic evaluations of adult male circumcision for prevention of heterosexual acquisition of HIV in men in sub-Saharan Africa: A systematic review," PLoS ONE 5(3): e9628. doi: 10.1371/journal.pone.0009628.

WHO and UNAIDS. 2011. "Joint strategic action framework to accelerate the scale-up of voluntary medical male circumcision for HIV prevention in eastern and southern Africa, 2012-2016." Geneva: UNAIDS. Available: http://www.unaids.org/sites/default/files/en/media/unaids/ contentassets/documents/unaidspublication/2011/JC2251_Action_Framework_circumcision_ en.pdf.

WHO-CHOICE. 2014. “Cost effectiveness and strategic planning." Available http://www.who.int/ choice/cost-effectiveness/inputs/capital_goods/en/

World Health Organization (WHO). 2015. "Progress brief: voluntary medical male circumcision for HIV prevention in 14 priority countries in East and Southern Africa." Available: http://www.who. int/hiv/pub/malecircumcision/brief2015/en/. 

Project SOAR

Population Council 4301 Connecticut Ave, NW,

Suite 280

Washington, D.C. 20008 USA

Tel: +1 2022379400

Fax: +1 2022378410

Ideas. Evidence. Impact.

projsoar.org 